\title{
Abscesso hepático por espinha de peixe: relato de caso
}

\author{
Hepatic abscess per fish spine: case report
}

Absceso hepático por espinas de pescado: relato de caso

\begin{abstract}
Hatsumi Miyashiro Iwamoto Meloํ․ Laiane Moraes Dias², Tanise Nazaré Maia Costa², Rafael Jose Romero Garcia ${ }^{3}$, Fernanda Oliveira Barreto Garcia ${ }^{3}$, Diego de Vasconcelos Melo4, Yuri Jose Almeida da Silva ${ }^{3}$, Carla Daniele Nascimento Pontes ${ }^{3 *}$, Rodrigo Bona Maneschy ${ }^{3}$, Lucianna Serfaty de Holanda $^{3}$, Vitor Bruno Teixeira de Holanda ${ }^{3}$, Lorena Faiz Storch Kuster Pantoja ${ }^{5}$, Weyce Mayane Cunha de Oliveira Baia ${ }^{6}$, Manoel Bastos Ribeiro Neto ${ }^{7}$.
\end{abstract}

\section{RESUMO}

Introdução: $\mathrm{O}$ abscesso hepático causado por corpo estranho é evento extremamente raro e de difícil reconhecimento, pois os exames de imagem dificilmente visualizam a perfuração, e os pacientes raramente relatam a ingestão de corpo estranho. Detalhamento do Caso: Paciente adulta iniciou de forma insidiosa quadro de dor abdominal, sendo atendida em diversas ocasiões em pronto-atendimento até receber diagnóstico ultrassonográfico de abscesso hepático. Foi encaminhada ao serviço de referência em hepatologia, onde recebeu tratamento com antibioticoterapia e abordagem cirúrgica com drenagem do abscesso, rafia do corpo gástrico e remoção do corpo estranho - uma espinha de peixe de 4 centímetros de comprimento. Paciente evoluiu sem intercorrências e recebeu alta para acompanhamento ambulatorial. Discussão: Abscessos hepáticos causados por perfuração gastrointestinal secundária a corpo estranho são potencialmente graves e há grande risco de mortalidade se não forem adequadamente tratados. O diagnóstico é feito preferencialmente por tomografia computadorizada de abdome e o tratamento de escolha é a combinação de antibioticoterapia e drenagem cirúrgica do abscesso.

Palavras-chave: Abscesso hepático, Abscesso hepático piogênico, Estranho, Corpos estranhos.

\begin{abstract}
Introduction: Hepatic abscess due to ingested foreign body is extremely rare and difficult to recognize, because radiological evaluations rarely detect perforation, and patients rarely report foreign body ingestion. Case Detail: A woman presented abdominal pain that progressively worsened, and was attended in the emergency room for multiple times, until she received an ultrasound diagnosis of liver abscess. After that, she was directed to the Hepatology Reference Service and started a treatment with antibiotic therapy and surgical approach with abscess drainage, gastric body raffia and removal of the foreign body - a $4 \mathrm{~cm}$ fishbone. The patient evolved with no complications and received hospital discharge for follow-up. Discussion: Hepatic abscess due to perforation of the gastrointestinal tract caused by ingested foreign bodys are potentially severe,
\end{abstract}

\footnotetext{
${ }^{1}$ Hospital Ophir Loyola, Belém-PA.

2 Centro Hospitalar Jean Bitar.

${ }^{3}$ Fundação Santa Casa de Misericórdia, Belém-PA. * E-mail: pontes4@live.com

${ }^{4}$ Secretaria Municipal de Saúde de Belém (SESMA), Belém-PA.

${ }^{5}$ Hospital Universitário João de Barros Barreto, Belém-PA.

${ }^{6}$ Hospital Porto Dias, Belém-PA.

${ }^{7}$ Fundação Hospital de Clínicas Gaspar Viana, Belém-PA.
} 
with a high risk of mortality if not properly treated. The diagnosis is preferably made with abdominal computed tomography, and the management is made with antibiotic therapy and surgical drainage of the abscess.

Key-words: Liver abscess, Liver abscess pyogenic, Foreign body, Foreign bodies.

\section{RESUMEN}

Introducción: El absceso hepático causado por un cuerpo extraño es un acontecimiento extremadamente raro y de difícil reconocimiento, ya que los exámenes de imagen no visualizan la perforación, y los pacientes raramente reportan la ingestión de un cuerpo extraño. Detalle del Caso: Paciente adulta inició de forma insidiosa cuadro de dolor abdominal, fue atendida en diversas ocasiones en atención a la atención hasta recibir diagnóstico ultrasonográfico de absceso hepáticoDespués de eso fue encaminada al servicio de referencia en hepatología donde recibió tratamiento con antibioticoterapia y estabilización clínica hasta que se realizó procedimiento quirúrgico con drenaje del absceso, rafia del cuerpo gástrico y remoción del cuerpo extraño - una espina de pescado de 4 centímetros de saludo. El paciente recibió un alto estable para el seguimiento ambulatorio. Discusión: Pacientes que forman absceso hepático por perforación gastrointestinal secundaria a cuerpo extraño son potencialmente graves y si no se adecuan adecuadamente hay un gran riesgo de mortalidad. El diagnóstico es preferentemente por tomografía computarizada de abdomen y el tratamiento de elección se combina con antibiótico y drenaje del absceso, ya sea por vía abierta o percutánea.

Palabras clave: Absceso hepático, Absceso piógeno hepático, Extraño, Cuerpos extraños.

\section{INTRODUÇÃO}

O abscesso hepático causado por corpo estranho é evento extremamente raro, com incidência menor de $1 \%$ e de difícil reconhecimento, pois os exames de imagem dificilmente visualizam a perfuração, e os pacientes raramente relatam a ingestão de corpo estranho (WAISBERG et al., 2002; GIGIREY et al., 2012; IGREJA et al., 2016).

As pessoas frequentemente ingerem corpos estranhos e eles geralmente atravessam o sistema gastrointestinal sem complicações. Perfuração intestinal e formação de abscessos ocorre ocasionalmente, e os sintomas podem surgir após meses a anos (SILVA et al., 2006). Os principais sítios de perfuração são o estômago e o duodeno, embora o local de penetração nem sempre possa ser identificado (CHONG et al., 2014). A relação entre o abscesso hepático no lobo esquerdo com a presença de corpo estranho é um desafio diagnóstico, e acarreta alta mortalidade. O corpo estranho geralmente consiste em espinha de peixe, ossos de galinha, agulhas ou palitos de dente, embora canetas ou placas dentárias já tenham sido relatadas (VEGA et al., 2001).

A apresentação clínica clássica de abscesso hepático inclui febre, dor abdominal e icterícia, porém é pouco frequente, e a maioria dos pacientes desenvolve sintomas pouco específicos como anorexia, vômitos, perda de peso, leucocitose, e elevação de transaminases ou fosfatase alcalina (LEE et al., 2005). Por isso é importante a realização de exames de imageamento como tomografia computadorizada e ultrassonografia de abdome (SILVA et al., 2006).

A aspiração percutânea com agulha fina guiada por exames de imagem auxilia no diagnóstico diferencial e pode determinar o agente responsável, caso trate-se realmente de um abscesso hepático (SILVA et al., 2006). Os microorganismos geralmente encontrados são bactérias entéricas Gram-negativas, particularmente a E. coli, K. pneumoniae, S. faecalis e P. vulgaris, e deve-se lembrar que a infecção por múltiplos organismos também é frequente (BORBA et al., 2012).

O tratamento do abscesso hepático inclui drenagem e antibioticoterapia. Porém, se há suspeita ou confirmação por exame de imagem de perfuração por corpo estranho, a cirurgia é o tratamento de escolha, apesar de haver relatos de remoção endoscópica ou percutânea (CHINTAMANI et al., 2003; LAMBERTUCCI 
et al, 2001). O tratamento clínico isolado apresenta mortalidade próxima a 100\% (SILVA et al., 2006). São considerados fatores de pior prognóstico: hemoglobina menor que $11 \mathrm{~g} / \mathrm{dll}$, BT maior que 1,5 mg/Ul, leucometria maior que 15.000 céls/mm3, albumina maior que 2,5 e TTPA elevado (SANTOS et al., 2007).

\section{DETALHAMENTO DO CASO}

Em 04 de novembro de 2017 foi internada na Fundação Santa casa de Misericórdia do Pará (FSCMPA), a paciente MCFL, 60 anos, sexo feminino, ex-empregada doméstica, natural e residente de Soure - Ilha do Marajó - Pará, referindo dor abdominal, vômitos e "abscesso hepático".

Apresentava história de febre constante, náuseas e vômitos, sudorese fria e gosto amargo na boca desde outubro de 2017, e referiu ter procurado atendimento médico em Soure, onde ficou em observação, porém não foram realizados exames de imagem devido indisponibilidade no local. A paciente, então, dirigiu-se à cidade de Belém - Pará, onde foi atendida em unidade de urgência e realizou USG abdominal no dia 25/10/17. O laudo do exame descrevia fígado de textura heterogênea devido infiltração gordurosa, lobo esquerdo apresentando área cística com debris, medindo $6 \times 6 \mathrm{~cm}$. Impressão diagnóstica de Esteatose hepática grau II e Abscesso hepático.

Foi encaminhada à FSCMPA - Serviço de Referência em Hepatologia no Estado do Pará. Na admissão, paciente mantinha quadro de náuseas e vômitos.

Entre os antecedentes pessoais, constavam nove gestações, com nove partos normais, negava HAS ou DM, e era ex-etilista e ex-tabagista.

No exame físico admissional, a paciente encontrava-se em bom estado geral, consciente e orientada, ausculta cardiopulmonar sem alterações, abdome flácido, ruídos hidroaéreos presentes, abaulamento em região epigástrica e extremidades com edema discreto.

Os exames laboratoriais da admissão apresentaram anemia discreta, leucocitose importante (20.900) e eletrólitos normais. Foi iniciado antibioticoterapia com Ceftriaxona e Metronidazol.

No segundo dia de internação, paciente foi avaliada pelo Grupo do Fígado - equipe de hepatologistas e cirurgiões especialistas em fígado e via biliar - que orientou a manutenção da antibioticoterapia, solicitou Tomografia de Abdome Total, Endoscopia Digestiva Alta, Marcadores tumorais e Sorologias.

Inicialmente foi mantida a antibioticoterapia devido resposta leucométrica e clínica satisfatórias. Os exames laboratoriais demonstraram marcadores tumorais normais, sorologias negativas para hepatites, e que a paciente não havia sido vacinada para hepatite $B$.

No $5^{\circ}$ dia de internação hospitalar, foi realizado Tomografia Computadorizada de Abdome, cujo laudo descrevia: Fígado de dimensões aumentadas, contornos lobulados e densidade heterogênea, às custas de imagens hipodensas confluentes, com densidade sólido-cística com pequeno foco gasoso em seu interior, de paredes espessas, limites parcialmente definidos, a maior ocupando quase a totalidade do lobo esquerdo, medindo cerca de 7,9 x 7,4 x 6,5 cm com volume de 197,5 cm3, com realce periférico após administração de contraste, a qual foi relacionada a abscesso hepático. Notou-se ainda imagem linear hiperdensa, na topografia de corpo gástrico que se estende ao lobo hepático esquerdo, medindo cerca de $4 \mathrm{~cm}$, sugerindo corpo estranho (espinha de peixe?).

Após discussões entre as equipes de cirurgia e clínica médica, optou-se por abordagem cirúrgica com drenagem do abscesso hepático e colecistectomia. Foram solicitados exames pré-operatórios e avaliação da cardiologia para risco cirúrgico. A antibioticoterapia foi mantida, com acompanhamento clínico rigoroso para minimizar os riscos durante o procedimento.

No dia 16/11/18, a paciente foi encaminhada ao bloco cirúrgico e submetida a Laparotomia Exploradora para drenagem de abscesso hepático, colecistectomia e sutura de corpo gástrico, local pelo qual houve passagem da espinha de peixe gerando perfuração gástrica e formação de coleção hepática. O procedimento ocorreu sem intercorrência. 
Figura 1 - Imagem do corpo estranho visto em tomografia computadorizada.

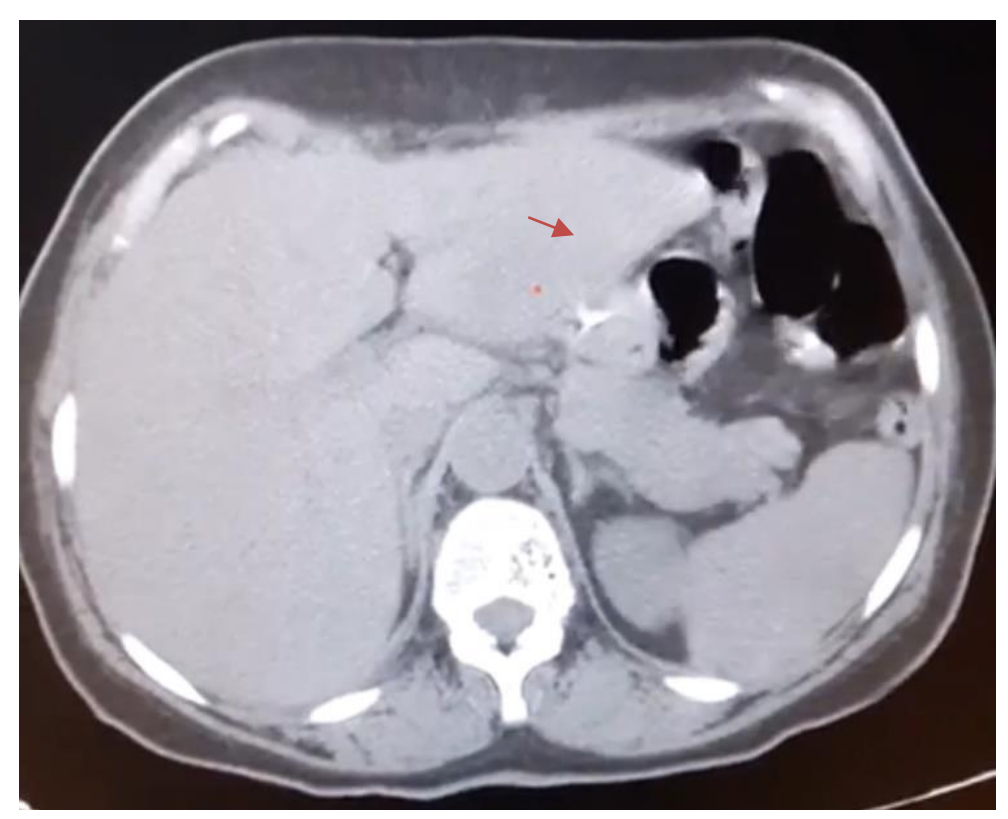

Fonte: Arquivo FSCMPA, 2017.

Figura 2 - Imagem do abscesso formado em fígado pela perfuração gástrica.

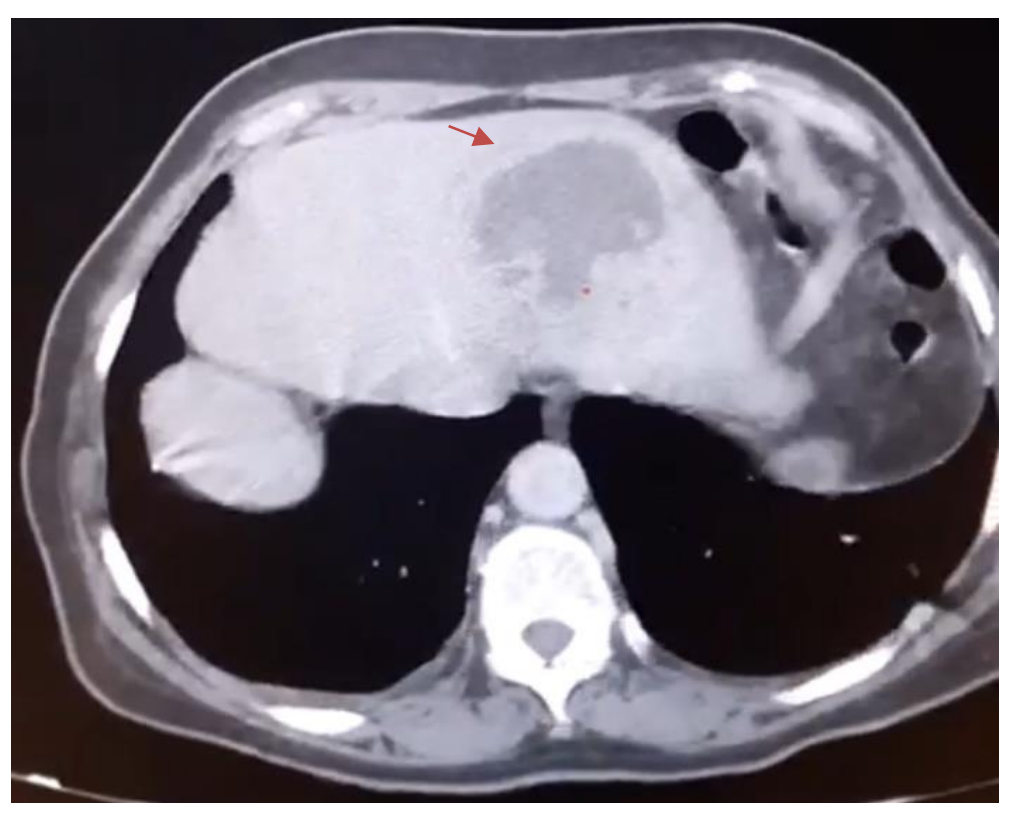

Fonte: Arquivo FSCMPA, 2017.

Após a cirurgia, a paciente foi encaminhada para seu leito de origem com analgesia, controle de diurese e suporte clínico. Optou-se por estender antibioticoterapia até completar 14 dias, e foi realizado tomografia computadorizada de abdome de controle para avaliação da drenagem e tamanho do abscesso. $O$ exame demonstrou resolução completa da lesão, a paciente manteve boa evolução clínica, e após 16 dias de internação recebeu alta melhorada para seguimento ambulatorial. 


\section{DISCUSSÃO}

A formação de abscesso hepático secundária à perfuração do trato gastrointestinal é evento raro, especialmente em localização gástrica. A maioria dos corpos estranhos ingeridos são impactados na região cricolaríngea e mais de $90 \%$ dos que conseguem passar pelo trato digestivo não causam nenhum tipo de agravo (SILVA et al., 2013).

O diagnóstico de abscesso hepático por corpo estranho é relativamente difícil devido a diversidade dos sintomas dependendo do grau de alteração que o corpo estranho pode causar, desde pequenas lesões até abdome agudo perfurativo. Devido a este fato, essa patologia não está na listagem dos primeiros diagnósticos diferenciais frente a um paciente com quadro de dor abdominal, epigastralgia ou mesmo náuseas e vômitos, sintomas estes que podem estar presentes (BORBA et al., 2012).

Os casos de abscessos hepáticos secundários a corpo estranho geralmente cursam de forma insidiosa com dor abdominal mal localizada, inespecífica, sem grandes sinais semiológicos positivos. Os sintomas constitucionais podem estar presentes como febre, astenia, e perda de peso devido à redução alimentar secundária a dor (MARCOLETA, 2010).

Por vezes, se não diagnosticados rapidamente, os abscessos hepáticos causados por corpo estranho podem ser fatais devido às suas complicações. A abordagem diagnóstica e terapêutica deve ser rápida e precisa, para que o paciente não sofra com as complicações e que tenha maior chance de sobrevida $(\mathrm{CHOI}$ et al, 2014).

Os exames de imagem são de fundamental importância para o diagnóstico de abscesso hepático. A Ultrassonografia de abdome ajuda a identificar coleções no parênquima hepático e definir quantidade de focos, mas a Tomografia Computadorizada com contraste é o método diagnóstico mais eficaz para delimitação precisa da extensão do abscesso, para localização e identificação do corpo estranho (MATRELLA et al., 2014).

As imagens visualizadas na tomografia computadorizada com contraste são em geral lesões únicas ou múltiplas, hipodensas, com realce periférico uniforme após contraste, com melhor visualização na fase tardia do mesmo, configurando um achado clássico de tecido de granulação. As margens podem ser nítidas, nos casos em que ocorre uma pseudo-cápsula, ou irregulares. Embora, esses achados não sejam patognomônicos e estejam classicamente presentes em apenas $35 \%$ dos abscessos, podem ajudar na diferenciação das leões hepáticas (SANTOS-ROSA et al., 2016).

O tratamento mais recomendado para os abscessos é a drenagem cirúrgica com remoção do corpo estranho e correção do local de perfuração, mas, em casos selecionados de perfuração gástrica mínima e aderência do estômago com o lobo hepático, pode ser utilizada uma técnica minimamente invasiva com drenagem percutânea ou endoscópica do abscesso com retirada endoscópica do corpo estranho. Ambas as técnicas são mais eficazes e seguras quando associadas a antibioticoterapia (CAl et al., 2015).

\section{REFERÊNCIAS}

1. Borba CC, Gomes ARS, Filgueira JPPS, et al. Abscessos hepáticos secundários a espinha de peixe. Relato de caso. Revista Brasileira de Clínica Médica, São Paulo, 2012; 10(1):83-6

2. Cai YL, Xiong XZ, Lu J, et al. Percutaneous needle aspiration versus catheter drainage in the management of liver abscess: a systematic review and meta-analysis. International Hepato-Pancreato-Biliary Association, China, 2015; 17(3):195-201.

3. Chintamani SV, Lubhana $\mathrm{P}$, Durkhere R, et al. Liver abscess secondary to a broken needle migration--a case report. BioMed Central Surgery, India; 2003; 3: 8.

4. Choi Y, Kim G, Shim C, et al. Peritonitis with small bowel perforation caused by a fish bone in a healthy patient. World Journal of Gastroenterology,South Corea,.2014; 20(6):1626-9.

5. Chong LW, Sun CK, Wu CC, et al. Successful treatment of liver abscess secondary to foreign body penetration of the alimentary tract: A case report and literature review. World Journal of Gastroenterology, 2014; 20(13): 37033711. 
6. Gigirey V, Parodi MR, Trapani ND et al. Absceso hepático secundario a cuerpo extraño - Presentacion de caso clínico y revision del tema. Revista de Imagenología, Uruguai, 2012; Vol. XVI, №1.

7. Igreja M, Cabral AL, Pensin L, et al. Abscesso hepático polimicrobiano por perfuração gástrica por corpo estranho. Polymicrobial hepatic abscess due to gastric perforation by foreign body. Revista da AMRIGS, Porto Alegre, 2016, 60 (4): 279-399.

8. Lambertucci JR, Rayes AA, Serufo JC, et al. Pyogenic abscesses and parasitic diseases. Rev Inst Med Trop São Paulo, 2001; 43(2):67-74.

9. Lee KF, Chu W, Wong SW, Lai PBS et al. Hepatic Abscess Secondary to Foreign Body Perforation of the Stomach. Division of Hepatobiliary and Pancreatic Surgery, Department of Surgery, Prince of Wales Hospital, The Chinese University of Hong Kong, Hong Kong, China, 2005; Asian Journal of Surgery, vol 28, $n^{\circ} 4$.

10. Marcoleta FBM. Absceso hepático: Enfrentamiento diagnóstico y terapêutico. Gastroenterología Latino Americana, 2010; 21(2):309-13.

11. Matrella F, Lhuaire M, Piardi T, et al. Liver hilar abscesses secondary to gastrointestinal perforation by ingested fish bones: surgical management of two cases. Hepatobiliary Surgery and Nutrition, 2014; 3(3): 156-162.

12. Santos SA, Alberto SCF, Cruz E, et al. Hepatic abscess induced by foreign body: Case report and literature review. World Journal of Gastroenterology, 2007; 13(9): 1466-1470.

13. Santos-Rosa OM, Lunardelli HS, Ribeiro-Junior MAF et al. Abscesso hepático piogênico: manejo dos recursos diagnósticos e terapêuticos ABCD Arq Bras Cir Dig, 2016; 29(3):194-197.

14. Silva RF, Arroyo Junior PC, Duca WJ, et al. Abscesso Hepático por Osso de Peixe Liver Abscess Caused by Fish Bone. Arq Ciênc Saúde 2006, 13(3):XX-XX.

15. Silva WLF, Indiani JMC, Martin MF et al. Qual o seu diagnóstico? Radiol Bras. 2013; 46(1):XV-XVI.

16. Vega M, Rivero JC, Ruíz L, et al. A fish bone in the liver. The Lancet 2001; Vol 358.

17. Waisberg J, Aráuz SNZ, Altieri LG et al. Abscesso hepático piogênico por corpo estranho. Rev. Col. Bras. Cir., 2002; Vol. 29, № 4. 